\title{
A clinical and multi-omics study of Van der Woude syndrome in three generations of a Chinese family
}

\author{
KAI YANG ${ }^{1 *}$, XING-YUE DONG ${ }^{2 *}$, JUE WU $^{3}$, JIAN-JIANG ZHU ${ }^{4}$, YA TAN $^{1}$, \\ YOU-SHENG YAN ${ }^{1}$, LI LIN ${ }^{1}$ and DONG-LIANG ZHANG ${ }^{2}$ \\ ${ }^{1}$ Department of Obstetrics and Gynecology, Peking University International Hospital, Beijing 102206; \\ ${ }^{2}$ Department of Orthodontics, School of Stomatology, Capital Medical University, Beijing 100050; ${ }^{3}$ Department of \\ Translational Medicine Laboratory, First Medical Center of People's Liberation Army General Hospital, Beijing 100039; \\ ${ }^{4}$ Department of Prenatal Diagnosis Center, Haidian Maternal and Child Health Care Hospital, Beijing 100080, P.R. China
}

Received January 23, 2020; Accepted June 23, 2020

DOI: $10.3892 / \mathrm{mmr} .2020 .11365$

\begin{abstract}
Previous studies have suggested that pathogenic variants in interferon regulatoryse factor 6 (IRF6) can account for almost $70 \%$ of familial Van der Woude Syndrome (VWS) cases. However, gene modifiers that account for the phenotypic variability of IRF6 in the context of VWS remain poorly characterized. The aim of this study was to report a family with VWS with variable expressivity and to identify the genetic cause. A 4-month-old boy initially presented with cleft palate and bilateral lower lip pits. Examination of his family history identified similar, albeit milder, clinical features in another four family members, including bilateral lower lip pits and/or hypodontia. Peripheral blood samples of eight members in this three-generation family were subsequently collected, and whole-exome sequencing was performed to detect pathogenic variants. A heterozygous missense $I R F 6$ variant with a c. $1198 \mathrm{C}>\mathrm{T}$ change in exon 9 (resulting in an R400W change at the amino acid level) was detected in five affected subjects, but not in the other three unaffected subjects. Moreover, subsequent structural analysis was indicative of damaged stability to the structure in the mutant IRF protein. Whole-transcriptome sequencing, expression analysis and Gene Ontology enrichment analysis were conducted on two groups of patients with phenotypic diversity from the same family. These analyses identified significant differentially expressed genes and enriched pathways in these two groups. Altogether, these findings provide insight into the mechanism underlying the variable expressivity of VWS.
\end{abstract}

Correspondence to: Professor Dong-Liang Zhang, Department of Orthodontics, School of Stomatology, Capital Medical University, 11 Xila Hutong, Dongcheng, Beijing 100050, P.R. China

E-mail: zhangdongliang@hotmail.com

${ }^{*}$ Contributed equally

Key words: Van der Woude syndrome, interferon regulatory factor 6 , cleft palate, whole-exome sequencing, whole-transcriptome sequencing

\section{Introduction}

Van der Woude Syndrome (VWS; OMIM. no. 119300, https://www.omim.org/) is a prevalent form of orofacial cleft syndrome, accounting for $2 \%$ of all cleft lip (CL) and cleft palate (CP) cases, and with an incidence of 1:35,000-1:100,000 worldwide $(1,2)$. Congenital lip pits, simultaneously occurring with $\mathrm{CL}$ and/or $\mathrm{CP}$, are the most common clinical feature of VWS, accounting for $80 \%$ of patients (3).

Numerous studies have demonstrated that pathogenic variants and polymorphisms in interferon regulatory factor 6 (IRF6; OMIM. no. 607199) contribute to the development of VWS (4-10). Although the gene modifier effect can explain the phenotypic variability of VWS, current understanding of the modifier genes of IRF6 remains incomplete and limited to a case-controlled genomic variant study (11). Additionally, the role of microRNA variants in the coding and non-coding regions of the genome in $\mathrm{CL}$ and $\mathrm{CP}$ pathogenesis has also been studied (12-15).

Advances in next generation sequencing have allowed the analyses of several diseases from a multi-omics perspective, particularly in oncology studies $(16,17)$. For instance, trio whole-exome sequencing (WES) is an established method for accurate detection of variants in genomic coding regions (18). Moreover, transcriptome sequencing has greatly facilitated gene expression studies (19). As a result, multi-omics analysis is increasingly applied to fundamental research and clinical diagnosis $(20,21)$.

The aim of the present study was to initially identify the genetic cause of VWS, and then to implement a multi-omic analysis in order to identify possible associations between different gene expression spectrums and phenotypic diversity in a three-generation family with VWS.

\section{Materials and methods}

Subjects. In September 2018, a three-generation Chinese family, including a 4-month-old boy with $\mathrm{CP}$, was recruited by the General Clinic of Beijing Stomatology Hospital, affiliated to Capital Medical University (Beijing, China). Routine physical examinations and a family history survey were 
conducted, with subsequent collection and storage of peripheral blood samples from eight family members, including the boy.

Chromosome karyotyping and chromosomal microarray analysis (CMA). Conventional karyotyping by G-banding, was performed on a blood sample from the proband boy according to standard operational procedures, in order to detect broad chromosomal anomalies. In addition, total genomic DNA $(1 \mu \mathrm{g})$ was extracted from $200 \mu \mathrm{l}$ peripheral blood using the DNA Blood Midi/Mini kit (Qiagen $\mathrm{GmbH}$ ), according to the manufacturer's protocols. A CMA was carried out with a CytoScan 750K SNP Array (Affymetrix; Thermo Fisher Scientific, Inc.), according to the manufacturer's protocol, in order to determine genomic copy number variants (CNV) with clinical significance based on the interpretation guideline (22). Data was collected and analyzed using GeneChip Scanner 3000 (Affymetrix; Thermo Fisher Scientific, Inc.) with Affymetrix GeneChip Convert Console (v.1.1) software (Affymetrix; Thermo Fisher Scientific, Inc.).

WES and data analysis. DNA samples $(1 \mu \mathrm{g})$ were extracted from blood samples $(200 \mu \mathrm{l})$ of all participants in the family using the DNA Blood Midi/Mini kit (Qiagen $\mathrm{GmbH}$ ), which then underwent quality control using agarose gel electrophoresis and UV spectrophotometry. Trio WES was carried out on the proband boy and his parents, as previously described (18). DNA fragments were hybridized and captured by xGen Exome Research Panel (Integrated DNA Technologies, Inc.), according to the manufacturer's protocol. The libraries were tested for enrichment by quantitative PCR, and the size, distribution and concentration were determined using an Agilent Bioanalyzer 2100 (Agilent Technologies, Inc.). The NovaSeq 6000 platform (Illumina, Inc.), along with 150 bp pair-end reads, was used for the genomic sequencing of DNA with $\sim 300$ pM per sample using NovaSeq Reagent kit.

Sequencing raw reads (quality level \%Q30 > 89\%) were aligned to the human reference genome (accession no. hg19/GRCh37; ftp://hgdownload.cse.ucsc.edu/goldenPath/hg19/chromosomes/) using the Burrows-Wheeler Aligner tool and the PCR duplicates were removed using Picard v1.57 (http://picard.sourceforge.net/). Variant calling was performed with the Verita Trekker ${ }^{\circledR}$ Variants Detection system (v2.0; Berry Genomics, Inc.) and Genome Analysis Toolkit (https://software.broadinstitute.org/gatk/). Variants were then annotated and interpreted using ANNOVAR (v2.0) and Enliven ${ }^{\circledR}$ Variants Annotation Interpretation systems (Berry Genomics, Inc.) (23), based on common guidelines by American College of Medical Genetics and Genomics (24). To validate variants, Sanger sequencing was introduced as a confirmatory method. Three-dimensional structure prediction was conducted with Modeller V9.21 (https://salilab. org/modeller/). The comparative modeling method with default parameters was used to construct the model based on the wild-type structure (PDB ID, 3DSHA; http://www.rcsb. org/structure/3DSH).

Whole-transcriptome sequencing and data analysis. RNA was then extracted from blood samples of five affected individuals and a healthy external control using Tempus ${ }^{\mathrm{TM}}$ Blood RNA
Tube and Tempus ${ }^{\mathrm{TM}}$ Spin RNA Isolation kit (Thermo Fisher Scientific, Inc.). RNA purity was checked by NanoPhotometer ${ }^{\circledR}$ spectrophotometer (Implen $\mathrm{GmbH}$ ). In addition, RNA integrity assessed using an RNA Nano 6000 assay kit on a Bioanalyzer 2100 system (Agilent Technologies, Inc.).

Sequencing libraries with sample indexing were generated using the NEBNext ${ }^{\circledR}$ Ultra $^{\mathrm{TM}}$ RNA Library Prep kit for Illumina (New England Biolabs, Inc.), according to the manufacturer's instructions. Briefly, mRNA was purified and a cDNA library was synthesized by reverse transcription, which was then fragmented into 250 300 bp. After PCR amplification, clustering was performed on the cBot Cluster Generation system, using a TruSeq PE Cluster kit v3-cBot-HS (cat. no. 401-3001; Illumina, Inc.). Finally, the library preparations, 2300 pM per sample, were sequenced on a HiSeq instrument (Illumina, Inc.), and $150 \mathrm{bp}$ paired-end reads were generated.

Raw reads were firstly processed to produce clean reads via the removal of reads containing adapters, reads containing ploy-N and low quality reads from raw data. At the same time, Q20, Q30 and GC content in the clean data were calculated. Then, data were mapped to the reference genome (accession no. hg19/GRCh37; ftp://hgdownload.cse.ucsc.edu/goldenPath/hg19/chromosomes/), quantification of gene expression levels was conducted using featureCounts v1.5.0-p3 (25). Differential expression analysis between two groups of patients, Group 1 and -2, was performed using the DESeq2 $\mathrm{R}$ package v1.16.1 (26) and genes with an adjusted $\mathrm{P}<0.05$ were considered differentially expressed (with the threshold: $\mid \log _{2}$ (fold-change) $>0.5$ ). In addition, Gene Ontology (GO) enrichment analysis of differentially expressed genes was performed by the clusterProfiler R package (27), in which gene length bias was corrected. GO terms with a corrected P-value $<0.05$ were considered significantly enriched by differentially expressed genes (28). The heatmap was generated using the pheatmap package (29).

\section{Results}

Clinical data. In this family, the affected patients included: i) III-1, a 4-month-old proband with $\mathrm{CP}$ and bilateral lower lip pits; ii) II-1, 38 years old with bilateral lower lip pits with gland (removed) and congenital hypodontia; iii) II-4, 37 years old with bilateral lower lip pits with gland (removed) and hypodontia; iv) II-7, 32 years old with bilateral lower lip pits; and v) I-2, 60 years old with bilateral lower lip pits. There are notable phenotypic heterogeneity among them, as demonstrated in Fig. 1 and Table I. The proband was the only patient with $\mathrm{CP}$, whereas the four adult patients also had differences in phenotypic severity, mainly in the presence of hypodontia.

Karyotyping and CMA. The karyotype of the boy was (46, XY), with no CNV with clinical significance detected by CMA (data not shown).

WES. Trio WES demonstrated that the proband boy carried a paternally inherited heterozygous $1198 \mathrm{C}>\mathrm{T}$ variant (translating to R400W at the amino acid level) in exon 9 of the IRF6 gene (Fig. 2A). Sanger sequencing also indicated all five affected individuals in this family (I-2, II-1, II-4, II-7 and III-1) shared the same variant. However, the remaining 
Table I. Clinical features of individuals from three generations of a family with Van der Woude syndrome.

\begin{tabular}{|c|c|c|c|}
\hline Individual & Age & Clinical features & Phenotype \\
\hline $\mathrm{I}-1$ & 32 years & Premature death from myocardial infarction & / \\
\hline $\mathrm{I}-2$ & 60 years & Bilateral lower lip pits & 1 \\
\hline II-1 & 38 years & $\begin{array}{l}\text { Bilateral lower lip pits with gland, removed by surgery at age } 5 \text {; congenitally } \\
\text { missing } 4 \text { teeth }\end{array}$ & 1,2 \\
\hline II-4 & 37 years & Bilateral lower lip pits with gland, removed by surgery at age 4 ; teeth not aligned & 1,2 \\
\hline II-7 & 32 years & Bilateral lower lip pits & 1 \\
\hline III-1 & 4 months & Bilateral lower lip pits; cleft palate & 1,3 \\
\hline III-3 & 4.5 years & Two buccal fistulas on neck, with surgical treatment & / \\
\hline III-4 & 7 years & $\begin{array}{l}\text { Currently in tooth replacement period; no other indications; tooth development to } \\
\text { be determined }\end{array}$ & I \\
\hline
\end{tabular}

Phenotypes are defined as 1, lip pits; 2, hypodontia; 3, cleft palate; /, unaffected.

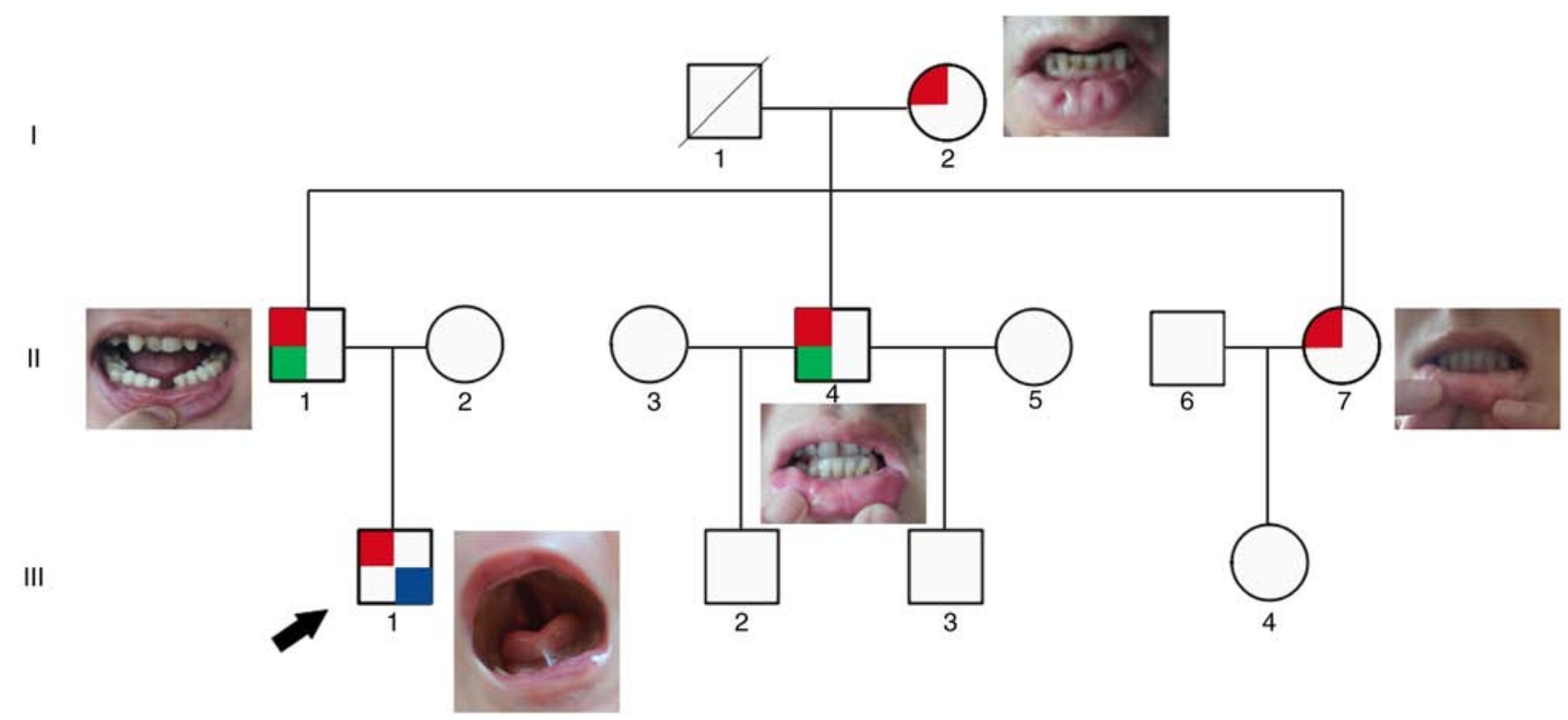

Figure 1. Pedigree of a three-generation Chinese family with Van der Woude syndrome and symptoms of affected patients. Red, lip pits; green, hypodontia; blue, cleft palate.

three unaffected individuals carried the wild-type IRF6 gene (Fig. 2A). Therefore, genetic examination demonstrated that variants and phenotypes in this family were co-segregated.

Structural prediction. The R400W variant turned a positively charged amino acid ( $\mathrm{R}$ ) into a polar amino acid with a benzene ring (W). Structural prediction suggested that, compared with the wild-type protein, the W400 residue in the mutant could not form hydrogen bonds and mediate electrostatic interactions with D227, thereby compromising overall structural stability (Fig. 2B and C).

Whole-transcriptome sequencing. The affected patients were divided into two groups according to phenotypic differences, mainly reflected in lip fistula tube formation and hypodontia. Group 1 included II-1 and II-4, whereas Group 2 consisted of I-2 and II-7. The four-month-old proband was excluded from the comparison due to the large age gap that could affect the results. To determine whether specific molecular pathways associated with IRF6 could account for the phenotypic differences between the two pairs, gene expression analysis was initially carried out. A volcano plot used to visualize the different expressing genes between the two groups. As presented in Fig. 3A, the red dots represent the upregulated genes (Group 1 vs. 2), whereas the blue dots represent the downregulated genes (Group 1 vs. 2).

In addition, the distribution of some significantly upregulated and downregulated expression genes is also presented in a heatmap (Fig. 3B). The horizontal coordinate was the sample, and the vertical coordinate was the differential gene; the left side clusters the gene according to the expression similarity degree, gene expression gradually upregulates from blue to red. The top 10 differentially expressed genes, other than those related to sex determination, are summarized in Table II. The results of GO enrichment analysis are displayed 


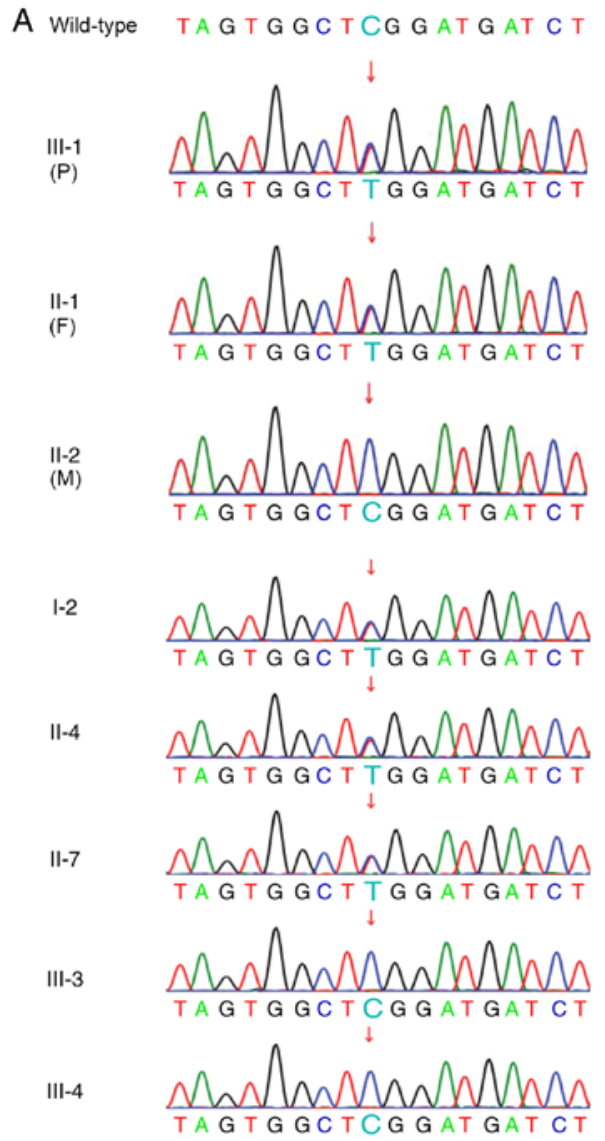

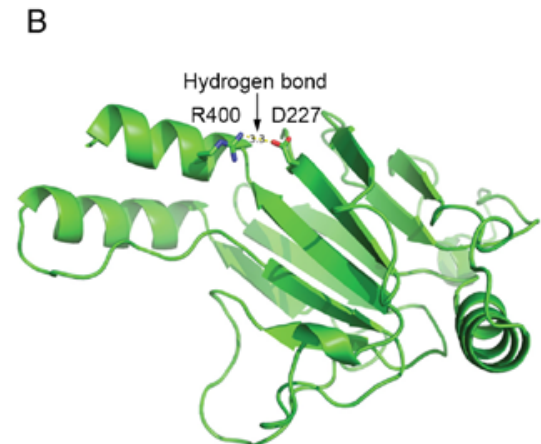

WT

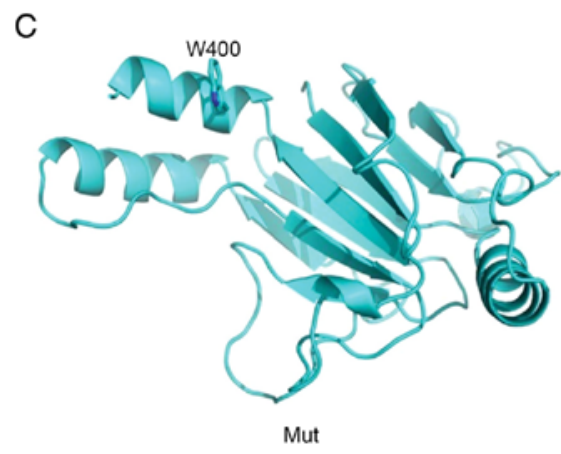

Figure 2. Identification of $1198 \mathrm{C}>\mathrm{T}$ (R400W) heterozygous variant in exon 9 of the IRF6 gene and structural prediction of the mutant protein. (A) Sanger sequences from all affected and unaffected individuals. Arrows indicate the variant site. (B) Structural prediction of the wild-type IRF6 protein, the hydrogen bond between R400 and D227 is indicated. (C) Structural prediction of the R400W mutant protein, demonstrating absence of hydrogen bonds and electrostatic interactions between W400 and D227 in the IRF6 protein structure. IRF6, interferon regulatory factor 6; P, proband; F, father; M, mother.

in Fig. 3C, which demonstrated that the most significantly expressed pathways between the two groups were associated with 'protein demethylation', 'protein dealkylation', 'histone lysine demethylation' and 'histone demethylation'.

\section{Discussion}

VWS is an autosomal dominant disorder, with patients usually presenting with CL and CP. Unlike non-syndromic CL and $\mathrm{CP}$, individuals with VWS exhibit bilateral and paramedian lower-lip fistulae, or occasionally, small mounds with a sinus tract deriving from a mucous gland of the lip (30). Moreover, hypodontia affecting maxillary incisors, canines and premolars also occurs in patients with VWS (15.5-86\%), together with other dental anomalies that may cause malocclusions (31). IRF6 gene mutations are commonly found in individuals with VWS (32).

The present study reported the case of a family with VWS spanning three generations. Five individuals (I-2, II-1, II-4, II-7 and III-1) were affected, with bilateral lower lip pits as the common symptom, and presented intrafamilial phenotypic variability, mainly in whether they displayed CP and hypodontia. WES suggested that all affected subjects carried a reported heterozygous variant c.1198C >T (R400W) of the IRF6 gene, whereas the unaffected did not (5). Protein structure analysis was carried out using prediction software, which suggested that this mutation might interrupt the formation of hydrogen bonds and electrostatic interactions between R400 and D227, thereby compromising overall structural stability. A previous study also described a recurrent c.1198C $>$ T $(\mathrm{R} 400 \mathrm{~W})$ mutation in IRF6 in Chinese families with VWS (7). According to the American College of Medical Genetics and Genomics criteria for variant interpretation (24), this variant has been determined as pathogenic (with evidence levels of PS1+PS2+PS4).

IRF6 contributes to the regulation of craniofacial development and epidermal proliferation (33). Moreover, numerous studies have indicated that variants in IRF6 that interrupt orofacial development are the main cause of VWS $(34,35)$. De Lima et al (32) carried out a sequencing analysis on 307 families with VWS and observed IRF6 exon variants in $68 \%$ of these, with $\sim 80 \%$ of IRF6 variants located in exons 3, 4, 7 and 9. Peyrard-Janvid et al (36) suggested that variants in IRF6 could account for $\sim 70 \%$ of familial cases of VWS, with a further $17 \%$ of patients resulting from grainy head like transcription factor 3 (GRHL3) variants.

IRF6 belongs to the IRF family of transcription factors and contains a highly conserved helix-turn-helix DNA-binding domain and a less conserved protein-binding domain. Most IRF6 variants are missense and result in protein truncation (37). The IRF family is composed of nine members, most of which are involved in mediating interferon responses following viral infection, as well as in innate immune responses (38). The IRF6 gene is $2,171 \mathrm{bp}$ in length and includes 10 exons, of which exons 1 , 
Table II. Top 10 genes (excluding gender-related genes) expressing significant differences between Group 1 (II-1 and II-4) and Group 2 (I-2 and II-7).

\begin{tabular}{|c|c|c|c|c|c|}
\hline Gene & Group 1, FKPM & Group 2, FPKM & $\log _{2}$ (fold-change) & P-value & P-adj \\
\hline ADARB2 & 0.02 & 4.53 & -6.37 & $2.73 \times 10^{-13}$ & $4.03 \times 10^{-12}$ \\
\hline TMEM176A & 1.67 & 6.92 & -1.77 & $1.09 \times 10^{-13}$ & $8.12 \times 10^{-11}$ \\
\hline PAM & 5.59 & 11.61 & -0.97 & $7.59 \times 10^{-12}$ & $5.31 \times 10^{-9}$ \\
\hline PRSS23 & 1.26 & 2.63 & -0.98 & $1.98 \times 10^{-11}$ & $1.34 \times 10^{-8}$ \\
\hline TMEM176B & 8.97 & 26.83 & -1.41 & $3.40 \times 10^{-11}$ & $2.19 \times 10^{-8}$ \\
\hline PLA2G7 & 3.13 & 0.79 & 1.69 & $2.98 \times 10^{-10}$ & $1.84 \times 10^{-7}$ \\
\hline MYBPH & 1.59 & 0.32 & 1.89 & $4.74 \times 10^{-10}$ & $2.82 \times 10^{-7}$ \\
\hline C20orf27 & 21.33 & 10.95 & 0.95 & $1.55 \times 10^{-9}$ & $8.55 \times 10^{-7}$ \\
\hline LGALS9B & 1.13 & 2.91 & -1.21 & $2.34 \times 10^{-9}$ & $1.24 \times 10^{-6}$ \\
\hline MYO7A & 0.64 & 0.26 & 1.22 & $1.29 \times 10^{-8}$ & $6.61 \times 10^{-6}$ \\
\hline
\end{tabular}

Fold-change was calculated as Group 1 standardized read counts/Group 2 standardized read counts. FKPM, fragments per kilobase per million mapped reads; P-adj, adjusted P-value.
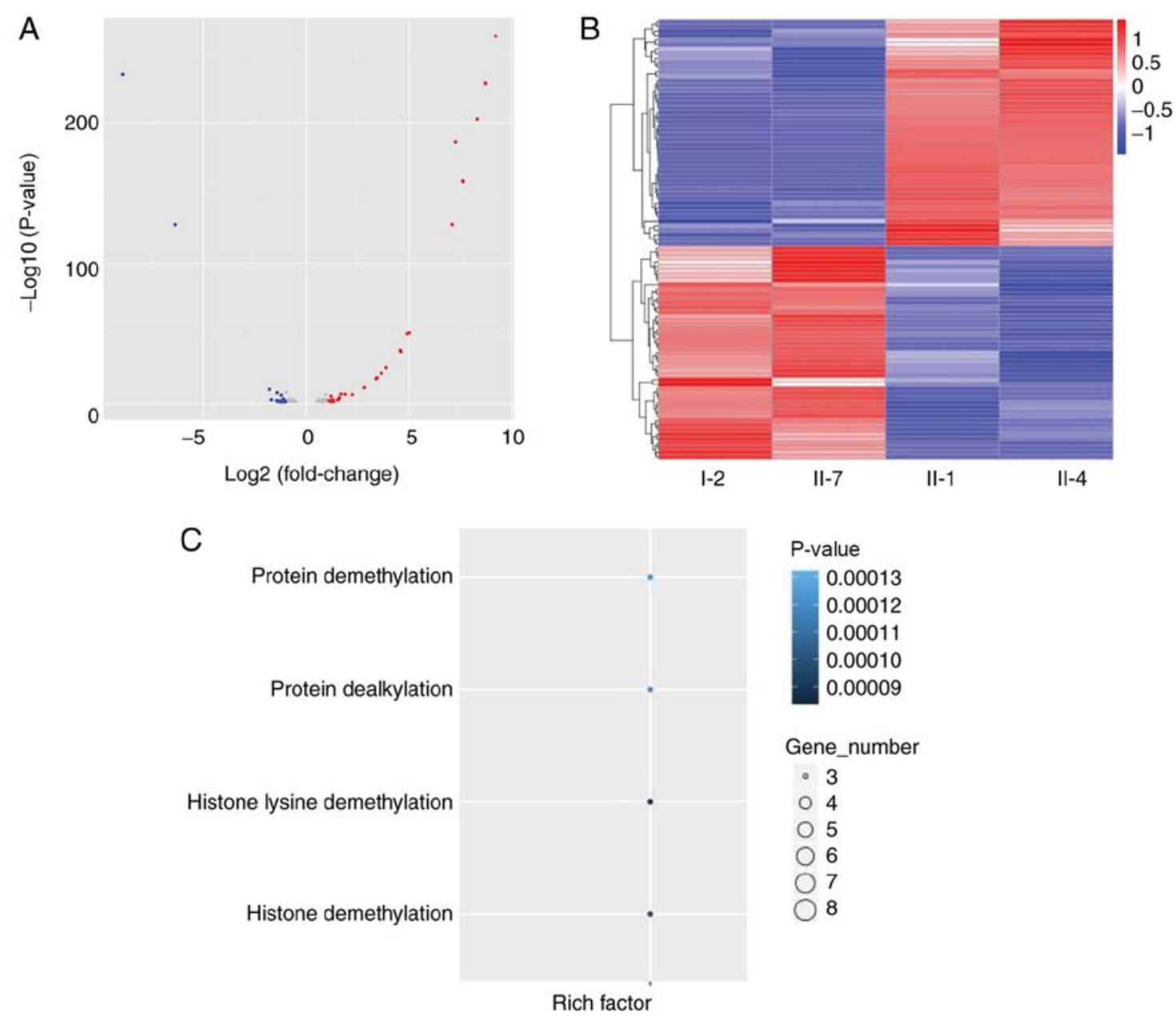

Figure 3. Differential expression analysis between patient group 1 and -2. (A) Volcano plot illustrating differentially expressed genes. X-axis represents $\log _{2}$-scaled fold-change; $y$-axis represents - $\log _{10}$-scaled P-values. Red and blue dots represent up- and downregulated genes, respectively (threshold: Ilog ${ }_{2}$-Scaled fold-changel>1) (B) Heat map representing distribution of differentially expressed genes. Red and blue scale represents up- and downregulated genes, respectively. (C) Gene Ontology enrichment analysis of differentially expressed genes between Group 1 and -2 .

2 and 10 are non-coding regions (39). The coding regions have a total length of 1,404 bp and encode 467 amino acids (37).
Specifically, exons 3 and 4 encode the DNA-binding domain, with exons 7 and 8 encoding the protein-binding domain of IRF6 (40). 
Phenotypic variability in VWS families with IRF6 variant occurs frequently (11). For instance, a previous study identified the pathogenic IRF6 variant $265 \mathrm{~A}>\mathrm{G}(\mathrm{K} 89 \mathrm{E})$ in three affected members. However, the newborn proband was diagnosed with popliteal pterygium syndrome (PPS), whereas the mother presented with classic VWS and the maternal grandfather had VWS with minor signs of PPS (30).

In the present study, the five patients with VWS displayed varying phenotypic severity. A gene expression analysis was therefore carried out on the four adult affected patients, who all lived in similar circumstances. Differentially expressed genes were identified between the two groups. Among these, myosin-binding protein H (OMIM. no. 160795) is expressed in a pattern specific to skeletal muscle (41). Myosin VII A (OMIM. no. 276903) is an unconventional myosin that is responsible for the formation and maintenance of epithelial cells in several human and animal organs (42). Adenosine deaminase RNA specific (ADAR) B2 (OMIM. no. 602065), which had the most significant expression difference between Group 1 and 2, is a member of the double-stranded RNA adenosine deaminase family of RNA-editing enzymes and can inhibit in vitro RNA editing by other ADAR family members (43). GO analysis suggested that these differentially expressed genes were associated with pathways related to protein and histone modifications. Whether these genes play a role in VWS phenotypic variability and interactions with IRF6 will require further study. Animal models may need to be developed and other 'omic' methods may have to be employed.

Furthermore, several previously reported genes related to bone formation, including Wnt family, fibroblast growth factor family, the Sonic hedgehog pathway, $\beta$-catenin, GRHL3, AP-2 complex subunit $\alpha$, receptor interacting serine/threonine kinase 4 , and TGF- $\beta$ activated kinase $1(33,36,44-46)$ were examined. There was no significant difference in expression in these genes between the two groups (Table SI), along with the results shown in Fig. 3, suggesting that VWS phenotypic variability may predominantly be associated with epigenetic levels and protein posttranslational modifications. However, this also requires further in-depth research, including investigation into epigenetic and protein modification (47), to confirm.

In conclusion, using WES, a heterozygous $1198 \mathrm{C}>\mathrm{T}$ IRF6 variant was identified in five affected members of a Chinese family with VWS. Protein structure prediction suggested overall structure stability was compromised in the R400W mutant protein. Gene expression and GO enrichment analysis also provided insight into a possible mechanism, such as epigenetic modification, to explain phenotypic variability in VWS. However, small sample size was a major limitation of this study. Therefore, a larger study sample is necessary, and further functional experiments are needed to validate the present findings.

\section{Acknowledgements}

Not applicable.

\section{Funding}

The present study was supported by The National Key Research and Development Program of China (grant no. 2017YFC1001700) and The Maternal and Child Development Special Program of Haidian Maternal and Child Healthcare Hospital (grant no. 201805).

\section{Availability of data and materials}

The datasets used and/or analyzed during the current study are available from the authors on reasonable request.

\section{Authors' contributions}

KY and DZ recruited subjects and designed the study. $\mathrm{KY}$ and $\mathrm{XD}$ wrote the manuscript, $\mathrm{XD}$ also analyzed the sequencing data. JZ conducted chromosomal karyotyping and chromosomal microarray analysis. JW and YT carried out whole-exome sequencing. YY and LL conducted the whole transcriptome sequencing and data analysis. All authors read and approved the final manuscript.

\section{Ethics approval and consent for participation}

Ethical approval was obtained from The Medical Ethics Committee of The School of Stomatology, Capital Medical University. All participants, or their guardians, signed written informed consent, including permission to publish anonymized test results.

\section{Patient consent for publication}

Consent was obtained from participants for the publication of this report and any accompanying images.

\section{Competing interests}

The authors declare that they have no competing interests.

\section{References}

1. Sudhakara Reddy R, Ramesh T, Vijayalaxmi N,Lavanya Reddy R, Swapna LA and Rajesh Singh T: Van der woude syndrome-a syndromic form of orofacial clefting. J Clin Exp Dent 4: e125-e128, 2012.

2. Angiero F, Farronato D, Ferrante F, Paglia M, Crippa R, Rufino L, Trevisiol A, Mazzola RF and Blasi S: Clinical, histomorphological and therapeutic features of the van der woude syndrome: Literature review and presentation of an unusual case. Eur J Paediatr Dent 19: 70-73, 2018.

3. Ural A, Bilgen F, Çakmakli S and Bekerecioğlu M: Van der woude syndrome with a novel mutation in the IRF6 gene. J Craniofac Surg 30: e465-e467, 2019.

4. Kwa MQ, Huynh J, Reynolds EC, Hamilton JA and Scholz GM: Disease-associated mutations in IRF6 and RIPK4 dysregulate their signalling functions. Cell Signal 27: 1509-1516, 2015.

5. Wang X, Liu J, Zhang H, Xiao M, Li J, Yang C, Lin X, Wu Z, $\mathrm{Hu} \mathrm{L}$ and Kong X: Novel mutations in the IRF6 gene for van der woude syndrome. Hum Genet 113: 382-386, 2003.

6. Ghassibe M, Bayet B, Revencu N, Verellen-Dumoulin C, Gillerot Y, Vanwijck R and Vikkula M: Interferon regulatory factor-6: A gene predisposing to isolated cleft lip with or without cleft palate in the Belgian population. Eur J Hum Genet 13: 1239-1242, 2005.

7. Ye XQ, Jin HX, Shi LS, Fan MW, Song GT, Fan HL and Bian Z: Identification of novel mutations of IRF6 gene in Chinese families with van der woude syndrome. Int J Mol Med 16: 851-856, 2005.

8. Du X, Tang W, Tian W, Li S, Li, X Liu L, Zheng X, Chen X, Lin Y and Tang Y: Novel IRF6 mutations in Chinese patients with van der woude syndrome. J Dent Res 85: 937-940, 2006. 
9. Khandelwal KD, Ishorst N, Zhou H, Ludwig KU, Venselaar $\mathrm{H}$, Gilissen C, Thonissen M, van Rooij IA, Dreesen K, Steehouwer M, et al: Novel IRF6 mutations detected in orofacial cleft patients by targeted massively parallel sequencing. J Dent Res 96: 179-185, 2017

10. Zhao H, Zhang M, Zhong W, Zhang J, Huang W, Zhang Y, Li W, Jia P, Zhang T, Liu Z, et al: A novel IRF6 mutation causing non-syndromic cleft lip with or without cleft palate in a pedigree. Mutagenesis 33: 195-202, 2018.

11. Leslie EJ, Mancuso JL, Schutte BC, Cooper ME, Durda KM, L'Heureux J, Zucchero TM, Marazita ML and Murray JC: Search for genetic modifiers of IRF6 and genotype-phenotype correlations in van der woude and popliteal pterygium syndromes. Am J Med Genet A 161: 2535-2544, 2013.

12. Kumari P, Singh SK and Raman R: A novel non-coding RNA within an intron of $\mathrm{CDH} 2$ and association of its SNP with non-syndromic cleft lip and palate. Gene 658: 123-128, 2018.

13. Gajera M, Desai N, Suzuki A, Li A, Zhang M, Jun G, Jia P, Zhao Z and Iwata J: MicroRNA-655-3p and microRNA-497-5p inhibit cell proliferation in cultured human lip cells through the regulation of genes related to human cleft lip. BMC Med Genomics 12: 70, 2019

14. Suzuki A, Abdallah N, Gajera M, Jun G, Jia P, Zhao Z and Iwata J: Genes and microRNAs associated with mouse cleft palate: A systematic review and bioinformatics analysis. Mech Dev 150: 21-27, 2018.

15. Thieme F and Ludwig KU: The role of noncoding genetic variation in isolated orofacial clefts. J Dent Res 96: 1238-1247, 2017.

16. Liu GM, Ji X, Lu TC, Duan LW, Jia WY, Liu Y, Sun ML and Luo YG: Comprehensive multi-omics analysis identified core molecular processes in esophageal cancer and revealed GNGT2 as a potential prognostic marker. World J Gastroenterol 25: 6890-6901, 2019.

17. Kiebish MA, Cullen J,Mishra P, Ali A, Milliman E, Rodrigues LO, Chen EY, Tolstikov V, Zhang L, Panagopoulos K, et al: Multi-Omic serum biomarkers for prognosis of disease progression in prostate cancer. J Transl Med 18: 10, 2020.

18. Yang K, Shen M, Yan Y, Tan Y, Zhang J, Wu J, Yang G, Li S, Wang J, Ren Z, et al: Genetic analysis in fetal skeletal dysplasias by trio whole-exome sequencing. BioMed Res Int 2019: 2492590, 2019

19. Jiang Z, Zhou X, Li R, Michal JJ, Zhang S, Dodson MV, Zhang $Z$ and Harland RM: Whole transcriptome analysis with sequencing: Methods, challenges and potential solutions. Cell Mol Life Sci 72: 3425-3439, 2015

20. Argelaguet R, Velten B, Arnol D, Dietrich S, Zenz T, Marioni JC, Buettner F, Huber W and Stegle O: Multi-Omics factor analysis-a framework for unsupervised integration of multi-omics data sets Mol Syst Biol 14: e8124, 2018.

21. Hasin Y, Seldin M and Lusis A: Multi-Omics approaches to disease. Genome Biol 18: 83, 2017.

22. Riggs ER, Andersen EF, Cherry AM, Kantarci S, Kearney H, Patel A, Raca G, Ritter DI, South ST, Thorland EC, et al: Technical standards for the interpretation and reporting of constitutional copy-number variants: A joint consensus recommendation of the American college of medical genetics and genomics (ACMG) and the clinical genome resource (ClinGen). Genet Med 22: 245-257, 2019.

23. Wang $\mathrm{K}, \mathrm{Li} \mathrm{M}$ and Hakonarson H: ANNOVAR: Functional annotation of genetic variants from next-generation sequencing data. Nucleic Acids Res 38: e164, 2010

24. Richards S, Aziz N, Bale S, Bick D, Das S, Gastier-Foster J, Grody WW, Hegde M, Lyon E, Spector E, et al: Standards and guidelines for the interpretation of sequence variants: A joint consensus recommendation of the American college of medical genetics and genomics and the association for molecular pathology. Genet Med 17: 405-424, 2015.

25. Zytnicki M: Mmquant: How to count multi-mapping reads? BMC Bioinformatics 18: 411, 2017.

26. Love MI, Huber W and Anders S: Moderated estimation of fold change and dispersion for RNA-seq data with DESeq2. Genome Biol 15: 550, 2014.

27. Yu G, Wang LG, Han Y and He QY: ClusterProfiler: An R package for comparing biological themes among gene clusters. OMICS 16: 284-287, 2012.

28. The Gene Ontology Consortium: The Gene Ontology Resource: 20 years and still GOing strong. Nucleic Acids Res 47: D330-D338, 2019

29. Zhang X, Yao X, Qin C, Luo P and Zhang J: Investigation of the molecular mechanisms underlying metastasis in prostate cancer by gene expression profiling. Exp Ther Med 12: 925-932, 2016
30. Busche A, Hehr U, Sieg P and Gillessen-Kaesbach G: Van der woude and popliteal pterygium syndromes: Broad intrafamilial variability in a three generation family with mutation in IRF6. Am J Med Genet A 170: 2404-2407, 2016.

31. Peralta-Mamani M, Terrero-Perez A, Dalben G, Rubira CMF, Honorio HM and Rubira-Bullen IF: Treatment of lower lip pits in van der woude syndrome: A systematic review. Int J Oral Maxillofac Surg 47: 421-427, 2018

32. de Lima RL, Hoper SA, Ghassibe M, Cooper ME, Rorick NK, Kondo S, Katz L, Marazita ML, Compton J, Bale S, et al: Prevalence and nonrandom distribution of exonic mutations in interferon regulatory factor 6 in 307 families with van der woude syndrome and 37 families with popliteal pterygium syndrome. Genet Med 11: 241-247, 2009

33. Oberbeck N, Pham VC, Webster JD, Reja R, Huang CS, Zhang Y, Roose-Girma M, Warming S, Li Q, Birnberg A, et al: The RIPK4-IRF6 signalling ax is safeguards epidermal differentiation and barrier function. Nature 574: 249-253, 2019.

34. Ingraham CR, Kinoshita A, Kondo S, Yang B, Sajan S, Trout KJ, Malik MI, Dunnwald M, Goudy SL, Lovett M, et al: Abnormal skin, limb and craniofacial morphogenesis in mice deficient for interferon regulatory factor 6 (Irf6). Nat Genet 38: 1335-1340, 2006.

35. Biggs LC, Naridze RL, DeMali KA, Lusche DF, Kuhl S, Soll DR, Schutte BC and Dunnwald M: Interferon regulatory factor 6 regulates keratinocyte migration. J Cell Sci 127: 2840-2848, 2014.

36. Peyrard-Janvid M, Leslie EJ, Kousa YA, Smith TL, Dunnwald M, Magnusson M, Lentz BA, Unneberg P, Fransson I, Koillinen HK, et al: Dominant mutations in GRHL3 cause van der woude syndrome and disrupt oral periderm development. Am J Hum Genet 94: 23-32, 2014.

37. Kondo S, Schutte BC, Richardson RJ, Bjork BC, Knight AS, Watanabe Y, Howard E, de Lima RL, Daack-Hirsch S, Sander A, et al: Mutations in IRF6 cause van der woude and popliteal pterygium syndromes. Nat Genet 32: 285-289, 2002.

38. Hixon K, Rhea L, Standley J, Canady FJ, Canady JW and Dunnwald M: Interferon regulatory factor 6 controls proliferation of keratinocytes from children with van der woude syndrome. Cleft Palate Craniofac J 54: 281-286, 2017.

39. Li S, Zhang X, Chen D, Zhao W, Zhang X, Jiao J, Guo L, Yin L, Song X, Liang C and Sun C: Association between genotype and phenotype of virulence gene in van der woude syndrome families. Mol Med Rep 17: 1241-1246, 2018.

40. Malik S, Wilcox ER and Naz S: Novel lip pit phenotypes and mutations of IRF6 in van der woude syndrome patients from Pakistan. Clin Genet 85: 487-491, 2014.

41. Vaughan KT, Weber FE, Ried T, Ward DC, Reinach FC and Fischiman DA: Human myosin-binding protein $\mathrm{H}(\mathrm{MyBP}-\mathrm{H})$ : Complete primary sequence, genomic orgnization, and chromosomal localization. Genomics 16: 34-40, 1993.

42. Bahloul A, Michel V, Hardelin JP, Nouaille S, Hoos S, Houdusse A, England P and Petit C: Cadherin-23, myosin VIIa and harmonin, encoded by usher syndrome type I genes, form a ternary complex and interact with membrane phospholipids. Hum Mol Genet 19: 3557-3565, 2010.

43. Chen CX, Cho DS, Wang Q, Lai F, Carter $\mathrm{kC}$ and Nishikura $\mathrm{K}$ A third member of the RNA-specific adenosine deaminase gene family, ADAR3, contains both single- and double-stranded RNA binding domains. RNA 6: 755-767, 2000.

44. Ke CY, Mei HH, Wong FH and Lo LJ: IRF6 and TAK1 coordinately promote the activation of HIPK2 to stimulate apoptosis during palate fusion. Sci Signal 12: eaav7666, 2019.

45. Maili L, Letra A, Silva R, Buchanan EP, Mulliken JB, Greives MR, Teichgraeber JF, Blackwell SJ, Ummer R, Weber R, et al: PBX-WNT-P63-IRF6 pathway in nonsyndromic cleft lip and palate. Birth Defects Res 112: 234-244, 2020.

46. Kousa YA, Fuller E and Schutte BC: IRF6 and AP2A interaction regulates epidermal development. J Invest Dermatol 138: 2578-2588, 2018

47. Sun Y, Weng Y, Zhang C, Liu Y, Kang C, Liu Z, Jing B, Zhang Q and Wang Z: Glycosylation of dentin matrix protein 1 is critical for osteogenesis. Sci Rep 5: 17518, 2015.

This work is licensed under a Creative Commons Attribution-NonCommercial-NoDerivatives 4.0 International (CC BY-NC-ND 4.0) License. 